\title{
Probing phonon propagation in materials by angle-resolved and angle-averaged vibrational EELS
}

\author{
Xingxu Yan ${ }^{1}$, Chaitanya Gadre ${ }^{2}$, Toshihiro Aoki ${ }^{3}$, Ruqian $\mathrm{Wu}^{4}$ and Xiaoqing Pan $^{2}$
}

${ }^{1}$ Department of Materials Science and Engineering, University of California - Irvine, Irvine, California, United States, ${ }^{2}$ Department of Physics and Astronomy, University of California, Irvine, CA 92697, Irvine, California, United States, ${ }^{3}$ Irvine Materials Research Institute, University of California, Irvine, Irvine, California, United States, ${ }^{4}$ Department of Physics and Astronomy, University of California, Irvine, CA 92697, United States

Since its establishment in 2015, UC Irvine Materials Research Institute (IMRI) has provided various state-ofthe-art materials research and characterization tools, including four TEMs to UCI and cross campus researchers, contributing to our recent NSF's Materials Research Science and Engineering Center (MRSEC) Award. Among the diverse and active research programs in UCI, vibrational EELS is one of the focused fields. Our efforts have been directed to how to apply the method to our materials research by utilizing Nion UltrasSTEM 200 HERMES equipped with Nion IRIS spectrometer, operating mostly at $60 \mathrm{keV}$ and occasionally at $30 \mathrm{keV}$ [1-2]. In-situ heating/biasing capabilities by Protochips Fusion allows us to conduct various in-situ studies [2].

Two different prove and EELS settings have been used in our vibrational EELS experiments. First one is the typical high resolution STEM probe where the convergence semi-angle is larger than 30mrad (more than a few angle settings exist). The second one is a small (3mrad) convergence semi-angle probe, adopted after Dwyer et al. [3]. The advantage of the large convergence semi-angle setting is that the probe is atomic size, thus atomic resolution STEM imaging and conventional core-loss EELS mapping are easily obtainable. Yet, because the convergence angle is larger than the first Brillouin Zone (FBZ), the vibrational signals observed are averaged over the entire FBZ. On the other hand, the small convergence semiangle probe makes it possible to attain diffraction patterns in which diffraction disks are discretely separated, allowing us to move EELS collection aperture to other than $\Gamma$ point with the angle resolution equal to full-convergence angle (Fig.1).

In this small convergence angle mode, the special resolution is limited to a few $\mathrm{nm}$ primarily by the diffraction limit. It is also found that the energy resolution gets slightly worsen due to an increased probe size at the sample, leading to a larger EELS entrance crossover [4]. The 3mrad probe gives us a good balance for the three key resolutions, angle (or momentum), spatial, and energy resolutions. Despite some small disadvantages, the small convergence semi angle probe brings angle or momentum resolution in vibrational EELS in STEM and thus has become a powerful tool in our research. The large and the small convergenceangle probe conditions can be called the angle (or momentum) averaged mode and the angle (or momentum) resolved mode.

Our recent research demonstrates single-defect phonons imaged by electron microscopy [5]. In the study, we detected a red shift of several $\mathrm{meV}$ in the energy of acoustic modes near a single stacking faults in cubic $\mathrm{SiC}$ by collecting EELS at X point, together with substantial changes in their intensity, found that these changes are confined to within a few nanometers of the stacking fault (Fig.2). It is demonstrated that angle-resolved mode allows us to identify small energy shift and change in intensity in vibrational mode at a single defect with high spatial resolution, opening the door to the direct mapping of phonon propagations around defects, which could not be possible by other techniques due to the limited spatial resolution. Currently the angle resolved/angle averaged vibrational EELS is being applied to study phonon propagations in SiGe quantum dots [1], [6].

We believe that this angle-resolved vibrational EELS, combined with angle-averaged mode is a powerful tool to help us understand and engineer thermal properties of materials. 

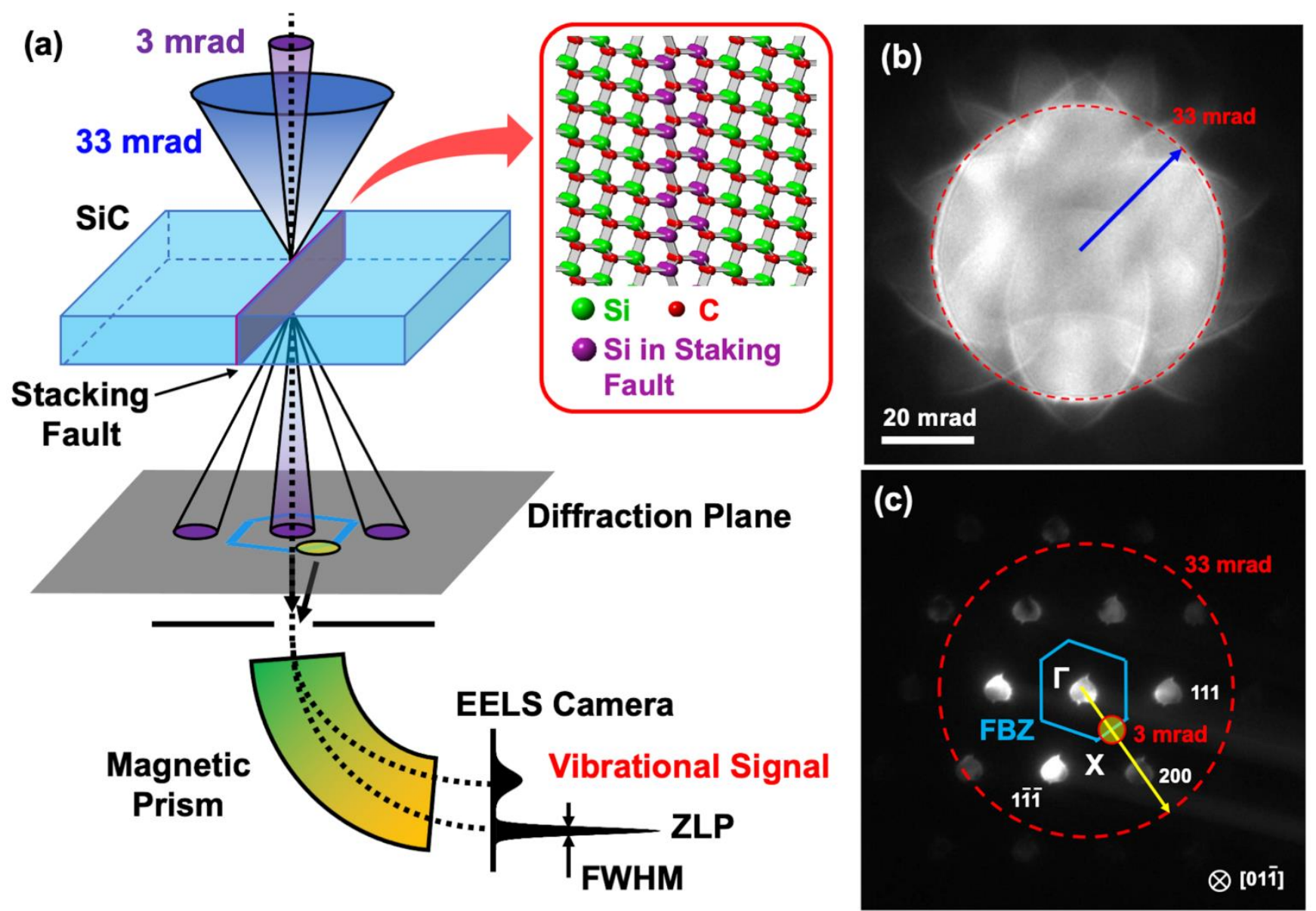

Figure 1. Figure 1. (a) Schematic of the experimental setup for our vibrational EELS [5]. The blue incident rays represent high resolution probe $(\alpha=33 \mathrm{mrad})$ and the purple rays represent the small convergence probe $(\alpha=3 \mathrm{mrad})$. Purple disks at the diffraction plane are the direct beam and the diffracted beams for 3mad. The yellow disk represents X point at the edge of FBZ. (b) diffraction pattern of SiC [01-1] for $\alpha=33 \mathrm{mrad}$; (c) diffraction pattern of SiC [01-1] for $\alpha=3 \mathrm{mrad}$, where the red dotted circle references $33 \mathrm{mrad}$ of convergence semi-angle, and the FBZ as blue contour and $\Gamma$ and $X$ point in FBZ.
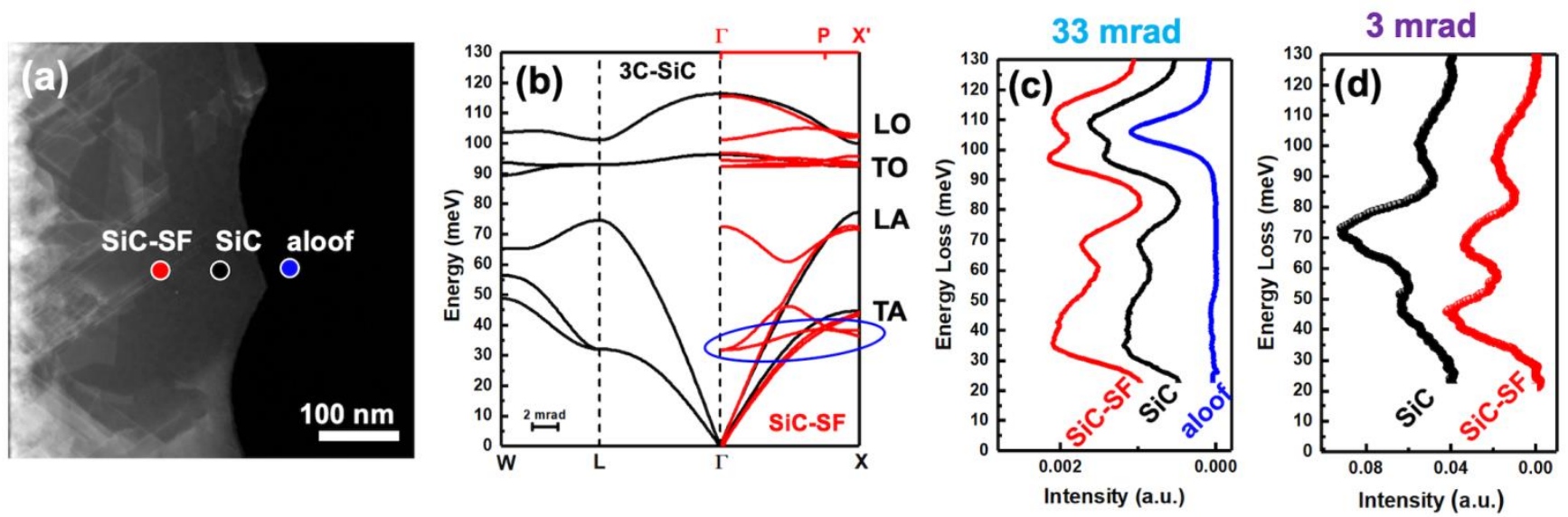

Figure 2. Figure 2. (a) Low-magnification medium-angle ADF-STEM image with marked electron beam locations for acquiring the aloof, $\mathrm{SiC}$ and $\mathrm{SiC}-\mathrm{SF}$ vibrational spectra, shown in (c). (b) 3C-SiC phonon dispersion curve showing where the angle-resolved vibrational EELS spectra were collected. (c) angle-averaged vibrational spectra of defect-free $\mathrm{SiC}$ (black curve), $\mathrm{SiC}$ with a stacking fault ( $\mathrm{SiC}-\mathrm{SF}$, red curve) and the aloof signal (blue curve) with a convergence 
semi-angle of $\alpha=33 \mathrm{mrad}$. (a). (d) Angle-resolved vibrational spectra of defect-free SiC (black) and of SiC with a stacking fault (red) with a convergence semi-angle of $\alpha=3 \mathrm{mrad}$ at the $\mathrm{X}$ point .

\section{References}

1. Gadre, C. et al., Microscopy and Microanalysis, 25(S2), 626-627(2019).

2. Yan, X. et al., Nano Lett. 19(10), 7494-7502(2019).

3. Dwyer, C. et al., Phys. Rev. Lett. 117, 256101(2016).

4. Krivanek, O. L. et al., Nature 514, 209-212(2014).

5. Yan, X., Liu, C., Gadre, C.A. et al., Nature 589, 65-69(2021).

6. Gadre, C. et al., Microscopy and Microanalysis 26(S2), 958-959(2020).

7. The authors acknowledge the use of facilities and instrumentation at the UC Irvine Materials Research Institute (IMRI) supported in part by the National Science Foundation Materials Research Science and Engineering Center program through the UC Irvine Center for Complex and Active Materials (DMR-2011967). 